\title{
A Complete Review and Comparative Study on Analysis of Data Clusters in Mining
}

\author{
Swati Vinodani \\ Galgotia College of Engineering
}

\author{
Aatif Jamshed \\ Assistant Professor Galgotia \\ College of Engineering
}

\author{
Pramod Kumar, PhD \\ Director \\ Tula`s Institute of Technology
}

\begin{abstract}
Clustering is a segment of data science into clusters of same items. Showing the data by less clusters essentially loses many fine points of interest, however accomplishes rearrangements. It shows data by the clusters. Data modeling places clustering in a verifiable pattern in data science, estimation, and numerical examination. As the machine learning is being considered so as to compare the clusters shrouded designs, the look of clusters is just like unsupervised learning, and the methodology says about the data idea. As the reasoning of the clusters is being considered which is taken as exception in the data mining, for eg, logical data analysis, data recovery and content mining, spatial database applications, Web examination, CRM, promoting, medicinal diagnostics, computational science, and numerous others. Clustering of data is being taken as the dynamic segment of many fields as estimation, design and artificial intelligence, which actually considers the clustering in the information science. Various type of the properties of the data points are considered for clustering the data points into the clusters. Some very meaningful algorithms are being used in the various clustering methodologies. The work in the paper provides the quick review of many clustering methodologies which works on various properties defined by the data points in the dataset.
\end{abstract}

\section{Keywords}

Clustering, Data Mining, Density, Learning, Distance, Similarity.

\section{INTRODUCTION}

Clustering is said to be major imperative inquiry of unsupervised machine learning, manages the data segment in obscure zone and is the reason for further mechanism. The entire working of the clustering is being defined not as fixed part but some of the considered facts of the methodologies [1]:

1) Points, in a similar cluster, are to be comparable however as more as expected;

2) Points, in the distinctive clusters, are to be diverse however much as could be expected;

3) Estimation for comparability and divergence should be clear and should have the reasonable significance;

The procedure of clustering can be isolated into the few steps[2]:

1) Feature extraction and determination: separate and pick major illustrative highlights from first data index;

2) Clustering algorithm: plan the clustering methodology as per the attributes of issue;

3) Result evaluation: assess the clustering output and consider the legitimacy of methodology;

4) Result evaluation: provide a useful clarification for the clustering output;

We are living in a world loaded with data. Consistently, peoples experience a lot of data and store or speak it as data, for facilitate analysis and management. One of the fundamental way in managing data is to order or clusters data into an arrangement of classifications or clusters. As a matter of fact, as a standout amongst the most crude exercises of peoples, classification plays a critical and basic part in the long history of human improvement. Considering the final outcome to take in another protest or comprehend another marvel, peoples dependably attempt to look for the features that can portray it, and further contrast it and other known items or wonders, in view of the similitude or uniqueness, summed up as nearness, as per some specific measures or standards. "Fundamentally, classification frameworks are either supervised or unsupervised, contingent upon whether they relegate new contributions to one of a limited number of discrete regulated classes or unsupervised classifications [3].

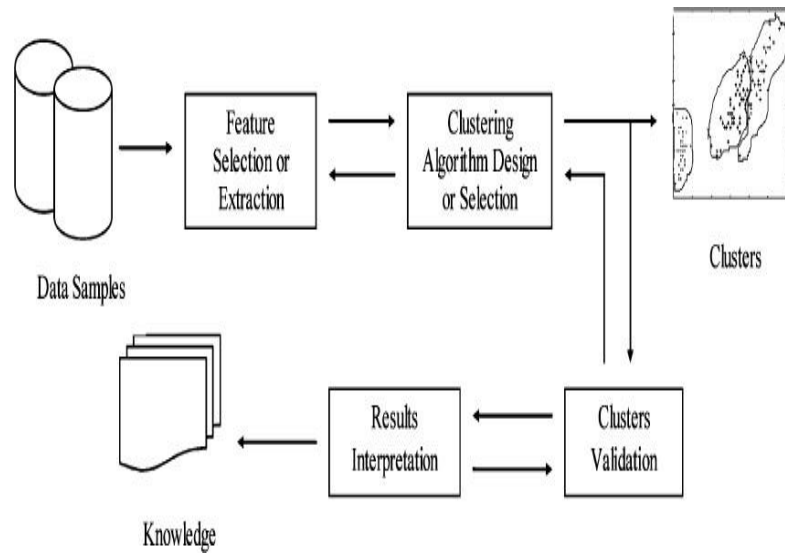

Fig 1: Clustering procedure.

In Clustering methodlogy the data is being divided in a specific number of clusters (clusters, subsets, or classifications). There exist no specific definition. Most analysts portray a cluster by taking the inward similarity and the outside division, i.e., designs in a similar cluster ought to be like one another, while designs in various clusters ought not. Both the similarity and the dissimilarity must be examinable in an unmistakable and important way. 


\section{CLUSTERING ALGORITHMS}

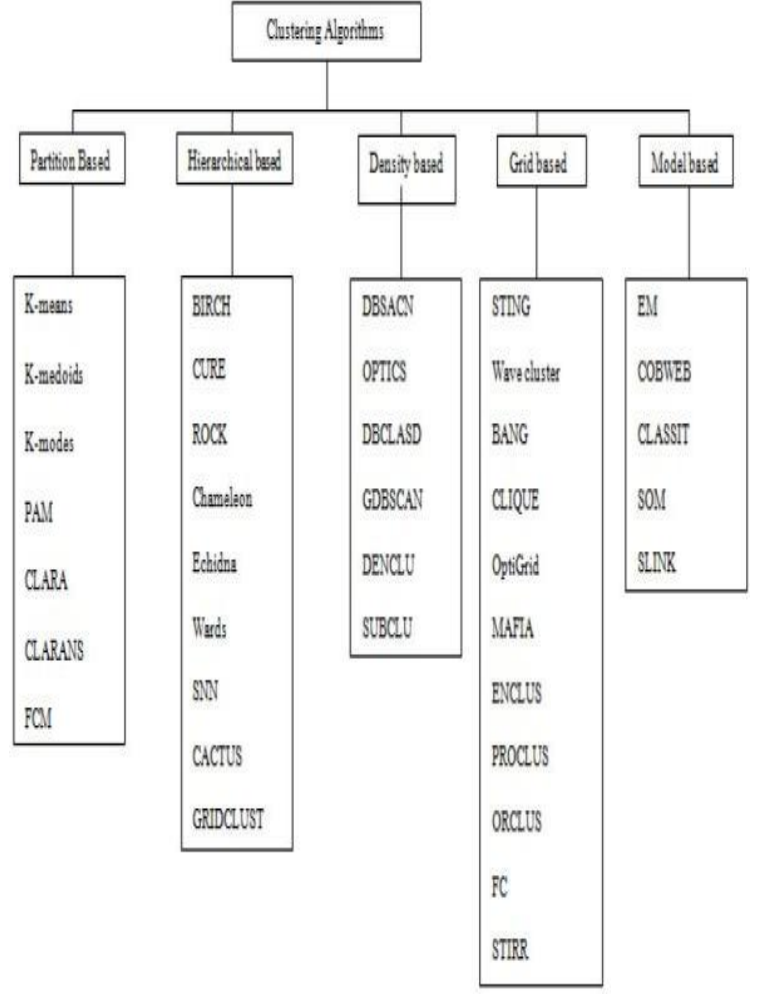

Fig 2: An overview of clustering algorithms for Big Data mining.

Clustering is the portioning of data into clusters of similar items [4]. The clustering methodology can be represented by five distinct classes, viz, Hierarchical, Partition, Spectral, Grid based and Density based clustering technique.

Hierarchical Clustering technique, the hierarchical clustering methodology is a cluster of data points framing a tree type pattern. It can be comprehensively clustered into agglomerative Hierarchical clustering and disruptive progressive clustering. In the agglomerative technique, which is additionally called as the bottom up approach, every data point is thought to be a different cluster, and on every step the clusters are combined, in view of a criteria. The clustering should be possible by utilizing the single connection, finish connection, centroid or wards technique. In the disruptive technique every data points are taken as a solitary cluster, and they are divided into various clusters, in light of specific criteria, and is termed as the top down technique. Cases of this algorithms are LEGCLUST, BRICH (Balance Iterative Reducing and Clustering utilizing Hierarchies), CURE (Cluster Using Rpresentatives), and Chemeleon.

The advantages of Hierarchical clustering incorporate inserted adaptability in regards to the level of granularity, and Easyness of treatment of any types of likeness or separation. Thusly, its relevance to any traits composes and its consistent pattern, make it simple to peruse and translate. The hindrances of Hierarchical clustering are identified with the unclearness of end criteria, the way that most progressive methodology don't return to once built, (moderate) clusters with a motivation behind their change; and which are generally unstable and unreliable, i.e., the main mix or detachment of articles, which might be founded on a little distinction in the paradigm, will oblige whatever is left of the analysis.
Spectral clustering is a kind of systems, which depends on the Eigen pattern of a similarity matrix. Clusters are framed by dividing data objects utilizing the similarity matrix. Any spectral clustering methodology have three primary steps [5]. They are preprocessing, spectral mapping and post mapping. Preprocessing manages the development of the similarity matrix. Spectral Mapping manages the development of Eigen vectors for the similarity matrix. Post Processing manages the clusters of data points.

The advantages of the Spectral clustering algorithm are: solid considerations on the cluster shape are not done:

- $\quad$ it is easy to implement;

- $\quad$ it doesn't select nearby optima;

- $\quad$ it is consistent and performs quicker.

The high computational complexity is one of the advantage of this technique. For huge database it considers $\mathrm{O}(\mathrm{n} 3)$, here $\mathrm{n}$ shows the data objects. Egs. of this technique are, SM(Shi and Malik) algorithm, KVV (Kannan, Vempala and Vetta) algorithm, and NJW ( Ng, Jordan and Weiss)algorithm

The grid based technique makes the data point space in a limited number of cells, that structures a matrix structure [6]. Tasks are performed on these matrices. The advantage of this technique is its low preparing time. Clustering complexity depends on the number of populated matrix cells, and does not rely upon the number of items in the dataset. The significant highlights of this technique are, no distance algorithms, Clustering is done on abridged data objects, Shapes are restricted to the association of grid cells, and the complexity of the methodology is typically $\mathrm{O}$ (Number of populated network cells).

The density based technique enables the offered cluster to keep on growing for more time as the Density in the next point surpasses a specific limit [7]. This technique is reasonable for taking care of noise in the database. The accompanying objects are listed as the highlights of this technique: it takes clusters of discretionary shape, Handles noise, needs just a single sweep of the data database, and the Density factors to be introduced. DBSCAN, DENCLUE and OPTICS are examples of this technique.

K-Means Clustering Algorithm is the clustering technique based on portioning of data points. It is advantageous as it is anything but difficult to execute and performs by considering the any of defined standards. It permits straight forward parallelization; and it is coldhearted concerning data requesting. The disadvantages of the $\mathrm{K}$ means technique are as per the following. The outcomes firmly rely upon the underlying supposition of the centroids. The neighborhood ideal (processed for a cluster) should not be a worldwide ideal (general clustering of an data index). It isn't evident what the great number $\mathrm{K}$ is for each situation, and the procedure is, as for the anomaly.

K-means maybe the mainstream clustering strategy in metric spaces. At first $\mathrm{k}$ cluster centroids[8] are chosen; $\mathrm{k}$ means then rearrange every one of the objects to the closest centroids and reevaluated centroids of the recently collected clusters. The iterative movement proceeds until the point when the paradigm work, e.g. square-error unites. Regardless of its wide fame, $\mathrm{k}$ - means is extremely delicate to noise and outlies since some of the data can considerably impact the centroids. Different shortcomings are affectability to initialization, captures into nearby optima, poor cluster descriptors, failure to manage clusters of discretionary shape, size and density, dependence on user to determine the number of clusters.

At last the technique considered the main objective as to minimize the objective function, considered the square error function for the above case which can be represented as: 


$$
j=\sum_{j=1}^{k} \sum_{t=1}^{n}\left\|x_{i}^{j}-c_{j}\right\|^{2}
$$

Fuzzy clustering [9] enables each element vector to have a place with in excess of one cluster with various participation degrees (0 and 1), and fuzzy outlines along the clusters. Fuzzy clustering is frequently utilized as a part of demonstrating (fuzzy modeling, neural systems, rule-based frameworks), where the clusters are looked for as a basic requirement for the following modeling exercises. For this situation, the perspectives can be shaped as a few points that are situated at the boundaries of the scope of data factors, with the goal that we accomplish an exhaustive "scope" of the data space, and thusly, the models "spread over" finished these data granules can be profoundly illustrative. The cluster display a specific "crowding" inclination. It is probably not going to see the clusters situated at the extraordinary estimations of the data factors and, hence, speak to these regions with regards to the development of the model.

To provide better representation on this impact in excess detail and represent its suggestions to framework demonstrating, let us consider a rule based model which is represented using principles of the shape if $\mathrm{x}$ is $\mathrm{Ai} \ldots$ at that point $\mathrm{y}$ is $\mathrm{Bi}$ and if $\mathrm{x}$ is $\mathrm{Ac} \ldots$ at that point $\mathrm{y}$ is $\mathrm{Bc}$, where $\mathrm{Ai}$ and $\mathrm{Bi}$ are fuzzy sets, which are characterized in data and output spaces, separately. Generally, these fuzzy sets are produced using fuzzy clustering, and the points of the clusters (models) are the methods of the fuzzy sets Ai and Bi. Implying the models framed through clustering, we consider that they are shaped in total input- output space, i.e., [vi mi]. Three challenges of the fuzzy clustering. First, the ideal number of clusters $\mathrm{K}$ to be made, must be determined (the number of clusters can't generally be characterized from the earlier and a good cluster legitimacy standard must be found). Second, the features and area of the cluster models (points) isn't really known from the earlier and beginning speculations must be made. Third, the data described by substantial fluctuation in the cluster shape, cluster density, and the number of objects (highlight vectors) in various clusters, must be dealt with.

Fuzzy C Means Clustering algorithm [10] considers the membership to every data object direct relating toward each cluster center, based on the separation for the data object and the center of the cluster. The data object which most nearest is to be taken as the center of the cluster, and also its membership will also more towards the cluster. Clearly, the addition of the membership of every data object ought to be equivalent to 1 . The benefits of the above defined technique is as it provides the better results for the covered collection of data, and performs good as compared to k- means algorithm. Not at all like the k-means, where the data point should only have a place with one cluster center, membership is being assigned to every data objects just because of the above fact the same data object may exist in more then a single cluster. The disadvantages of the clustering technique are, Apriori determination of the number of clusters; with a reduced estimation of $\beta$ improve result however to the detriment of more number of cycles and the Euclidean distance estimation can weight the elements unequally.

Genetic K-Means Algorithm [11] is a hybrid genetic technique (GA) that provides an internationally ideal segment of a provided data in a predefined number of clusters. GAs utilized before in clustering, utilize either a costly hybrid administrator to create substantial child chromosomes from parent chromosomes, or an expensive wellness work or both. To go around these costly activities, they hybridized the GA with a classical gradient descent technique utilized as a part of clustering, viz., the K-means technique. They characterized the K-means algorithm, one- advance of the K-means technique, and utilized it in GKA as a search operator, rather than hybrid. They likewise characterized a one-sided change operator particular to clustering, termed as distance based-mutation. Utilizing the finite Markov pattern hypothesis, they demonstrated that the GKA merges to a worldwide ideal. It is seen in the simulations that the GKA focalizes is ideal, comparing to the provided data, in simultaneousness with the union outcome. It is likewise watched that the GKA looks speedier than a portion of the other developmental techniques utilized for clustering. The advantage of GKA clustering technique is as it is quicker than a portion of the other clustering techniques.

CLARANS joins the sampling technique with PAM. The clustering procedure may be exhibited as looking through a chart where each hub is a best output, which is a set of k-medoids. The clustering got by interchanging a medoid is known as the neighbor of the present clustering. CLARANS [13] picks a node and thinks about it to a pre assigned neighbors hunting down slowly. If a superior neighbor is seen with less square mistake, CLARANS shifts to next node and procedure begins once more; generally the present clustering is a local optimum. If the local optimum is discovered, CLARANS begins with another randomly chosen node in search for another local optimum.

The advantages and disadvantages of partitioning clustering methods are:

\section{Disadvantages}

1) When high dimensional space is considered then it provides effective degradation as the object pairs are far away,

2) inefficient cluster descriptors,

3) Ask user to define the cluster number in prior,

4) High sensitivity to starting step, noise and outliers

5) Frequent entrapments into local optima

DBSCAN [14] looks for center points whose area (span) is in Minpts. An arrangement of center articles with covering neighborhoods characterize the pattern of a cluster. Non-center objects within the area of center object speak to the limits of the clusters, while all other are noise.

DBSCAN can find arbitrary formed clusters, is harsh to exceptions and request of data provided, and the complexity is $\mathrm{O}(\mathrm{N} 2)$. If a spatial list dataset is utilized the complexity can be enhanced to $\mathrm{O}(\mathrm{N} \log \mathrm{N})$. DBSCAN separates in high dimensional spaces and is exceptionally sensitive to the info parameters and Minpts.

Density based Clustering (DENCLUE) utilizes an impact capacity to portray the effect of a point about its next object while the general Density of the data objects is the aggregate of impact capacities from all points of data. Clusters are resolved utilizing Density attractors, nearby maxima of the general Density work. To process the sum of impact works a lattice structure is utilized. DENCLUE scales well $(\mathrm{O}(\mathrm{N}))$, can discover subjective molded clusters, is arbitrary safe, is obtuse to the data requesting, yet experiences its affectability to the data parameters. The curse of dimensionality wonder vigorously influences Denclue's viability. Besides, like Hierarchical and partitioning strategies, the output, marked points with cluster identifier, of Density based techniques cannot be effortlessly absorbed by people.

\section{Disadvantages}

- It is highly sensitive in the case of considering the parameters for input,

- $\quad$ Degraded cluster descriptors 
- Relevant for high-dimensional databases.

\section{COMPARATIVE ANALYSIS}

The paper comprises of several clustering algorithms which uses different parameters for clustering forming. The survey has all types of methodologies with different framework, this module of the paper will provide a brief comparison of the methodologies on the basis of certain parameters like complexity, input used, efficiency, etc.

Table 1. Comparative Study of different methodologies discussed for clustering the dataset.

\begin{tabular}{|c|c|c|c|c|c|}
\hline Clustering Algorithm & Data Size & Avoid Outliers & Dataset Type & Cluster Shape & Time Complexity \\
\hline K-Means Clustering & Large & No & Numerical & Non-Convex & $\mathrm{O}(\mathrm{nkd})$ \\
\hline Fuzzy Clustering & Large & Yes & Numerical & Arbitrary & $\mathrm{O}(\mathrm{n})$ \\
\hline Fuzzy C-means Clustering & Large & No & Numerical & - & $\mathrm{O}(\mathrm{n})$ \\
\hline CLARANS & Large & No & Numerical & Non-Convex & $\mathrm{O}\left(\mathrm{kn}^{2}\right)$ \\
\hline DBSCAN & Large & No & Numerical & Arbitrary & $\mathrm{O}(\mathrm{nlog} n)$ \\
\hline DENCLUE & Large & Yes & Numerical & Arbitrary & $\mathrm{O}(\log |\mathrm{D}|)$ \\
\hline OPTIC & Large & Yes & Numerical & Arbitrary & $\mathrm{O}(\mathrm{nlog} n)$ \\
\hline BRICH & Large & No & Numerical & Non-Convex & $\mathrm{O}(\mathrm{n})$ \\
\hline CURE & Large & Yes & $\begin{array}{l}\text { Numerical an } \\
\text { categorical }\end{array}$ & Arbitrary & $O\left(n^{2} \log n\right)$ \\
\hline
\end{tabular}

\section{CONCLUSION AND FUTURE DIRECTION}

As per the survey presented in the above work it is very much clear that the efficiency of the data points included in the cluster is still untouched. As most of the works presented the presentation of the data and also some has considered the preprocessing of the data before deciding which point is to be included into the specific cluster. The major problem that is missing is the similarity measure of the data points from the dataset to include it into the specific cluster. The problem of accuracy and redundancy of the dissimilar points in the clusters remains in the k-means and other variants of k-means algorithm, for which new enhanced approach is to be proposed which can work in better way to improve the efficiency of the lusters in terms of the similarity of the cluster data points included. In this work a brief description of the major clustering technique is being provided. The paper contains the proper definition of the technique, clustering requirements and also a brief detailing about the different types of clustering technique is being presented. The complete work roams around the data mining and its concepts along with definition of the required basic terminology in the technique. Then classification of the clustering technique discussed are the soft and the hierarchal technique for clustering of the data is being provided.

\section{REFERENCES}

[1] Jain A, Dubes R. 1988 Algorithms for clustering data. Prentice-Hall. Inc. Upper Saddle River.

[2] Xu R, Wunsch D. 2005. Survey of clustering algorithms. IEEE Trans Neural Network. 16. 645-678

[3] Velmurugan T, Santhanam T. 2011. A survey of partition based clustering algorithms in data mining: an experimental approach. Inf Technol J. 10.478-484.

[4] Ji He,Man Lan, Chew-Lim Tan, Sam-Yuan Sung, HweeBoon Low, "Initialization of Cluster refinement algorithms: a review and comparative study", Proceeding of International Joint Conference on Neural Networks [C]. Budapest, 2004.

[5] He, Z., Xu, X. and Deng, S. Scalable algorithms for clustering large datasets with mixed type attributes. International Journal of Intelligence Systems. 20. 10771089 
[6] Jiawei Han and Michheline Kamber. Data mining concepts and techniques. a reference book. pg. no.-383422.

[7] P. Arabie, L. J. Hubert, and G. De Soete. Clustering and Classification. World Scietific, 1996.

[8] Biswas, G., Weingberg, J. and Fisher, D.H.. ITERATE: A conceptual clustering algorithm for data mining. IEEE Transactions on Systems, Man, and Cybernetics. v28C. 219-230.

[9] R. Davé and R. Krishnapuram, "Robust clustering methods: A unified view," IEEE Trans. Fuzzy Syst., vol. 5, no. 2, pp. 270-293, May 1997.

[10] A. Geva, "Hierarchical unsupervised fuzzy clustering," IEEE Trans. Fuzzy Syst., vol. 7, no. 6, pp. 723-733, Dec.
1999.

[11] S. Bandyopadhyay and U. Maulik, "Nonparametric genetic clustering: Comparison of validity indices," IEEE Trans. Syst., Man, Cybern. C, Appl. Rev., vol. 31, no. 1, pp. $120-125$, Feb. 2001

[12] Chengjie GU, Shunyi ZHANG, Kai LIU, He Huang, "Fuzzy Kernal K-Means Clustering Method Based on Immune Genetic Algorithm", Journal of Computational Information Systems, Vol. 7, No. 1, pp. 221-231, 2011.

[13] A. Jain and R. Dubes, Algorithms for Clustering Data. Englewood Cliffs, NJ: Prentice-Hall, 1988.

[14] Jiawei Han, Micheline Kamber, "Data Mining Concepts and Techniques" Elsevier Publication. 